\title{
Guide de présentation des rapports d'examens systématiques
}

\author{
Correspondance : ccdr-rmtc@phac-aspc.gc.ca
}

Les examens systématiques résument l'état des connaissances à propos d'un sujet. Ils précisent à la fois les éléments connus et les éléments qui doivent faire l'objet d'études plus approfondies, et ils sont utilisés pour rester à jour, pour guider l'élaboration d'avis et de lignes directrices relatives aux pratiques cliniques, et pour déterminer les priorités pour les futures recherches. En général, ils ont une longueur de 2000 à 2500 mots - excluant le résumé, les tableaux et les références.

Le Relevé des maladies transmissibles au Canada (RMTC) soutient les lignes directrices PRISMA (Preferred Reporting Items of Systematic reviews and Meta-Analyses), largement acceptées (1). Le présent guide a été initialement mis au point pour les interventions en matière de soins de santé, et il a été adapté pour d'autres utilisations $(2,3,4)$.

Le Tableau 1 fournit la liste de vérification PRISMA. La Figure 1 illustre un organigramme qui détermine la façon dont le nombre initial d'études recensées durant une recherche documentaire a été réduit à l'essentiel pour les études aux fins d'examen.

Il y a d'autres facteurs à prendre en considération pour les examens systématiques sur les maladies infectieuses. Ceux-ci comprennent la nécessité de tenir compte des différences entre les études dans les méthodes de laboratoire utilisées pour la détermination des maladies infectieuses, de la présence ou du degré de résistance aux antibiotiques, et de la façon dont les définitions de cas ont été utilisées pour interpréter les résultats des analyses de laboratoire. On utilise des noms génériques pour désigner les antibiotiques ou les vaccins, et les noms de marques peuvent être indiqués entre parenthèses à la première utilisation.

Comme pour toutes les soumissions, vérifier les Renseignements à l'intention des auteurs (publiés en janvier chaque année avec le premier numéro de chaque nouveau volume) du Relevé des maladies transmissibles au Canada (RMTC) pour les exigences générales relatives à la préparation et à la soumission des manuscrits.

Tableau 1 : Liste de vérification PRISMA pour les examens systématiques ${ }^{1}$

\begin{tabular}{|c|c|c|}
\hline $\begin{array}{l}\text { Élément de la } \\
\text { déclaration }\end{array}$ & $\mathbf{N}^{01}$ & Description \\
\hline \multicolumn{3}{|l|}{ Titre } \\
\hline Titre & 1 & $\begin{array}{l}\text { Désigner le rapport en tant qu'examen systématique, en tant que } \\
\text { méta-analyse, ou les deux. }\end{array}$ \\
\hline \multicolumn{3}{|l|}{ Résumé } \\
\hline Résumé structuré & 2 & $\begin{array}{l}\text { Fournir un résumé structuré comprenant les sous-titres suivants : } \\
\text { Contexte, Objectifs; Sources de données; Sélection de l'étude, } \\
\text { Synthèse; Conclusion, et le numéro d'enregistrement de l'examen } \\
\text { systématique, le cas échéant. }\end{array}$ \\
\hline \multicolumn{3}{|l|}{ Introduction } \\
\hline Justification & 3 & $\begin{array}{l}\text { Décrire la justification de l'examen dans le contexte des faits déjà } \\
\text { connus. }\end{array}$ \\
\hline Objectifs & 4 & $\begin{array}{l}\text { Fournir un énoncé explicite de questions abordées par rapport aux } \\
\text { participants, des interventions, des comparaisons, des résultats et de } \\
\text { la conception de l'étude [p. ex. le projet PICOS (Privacy and Identity } \\
\text { Management for Community Services]. }\end{array}$ \\
\hline \multicolumn{3}{|l|}{ Méthodologie } \\
\hline
\end{tabular}




\begin{tabular}{|c|c|c|}
\hline $\begin{array}{l}\text { Protocole et } \\
\text { enregistrement }\end{array}$ & 5 & $\begin{array}{l}\text { Indiquer si un protocole d'examen existe, si et là où il est accessible } \\
\text { (p. ex. l'adresse du site Web), et s'ils sont disponibles, fournir des } \\
\text { renseignements sur l'enregistrement, y compris le numéro } \\
\text { d'enregistrement. }\end{array}$ \\
\hline Critères d'admissibilité & 6 & $\begin{array}{l}\text { Préciser les caractéristiques de l'étude (p. ex. le projet PICOS, la } \\
\text { durée et le suivi) et les caractéristiques du rapport (p. ex. les années } \\
\text { prises en considération, la langue, l'état de la publication) utilisées } \\
\text { comme critères d'admissibilité, en donnant une justification. }\end{array}$ \\
\hline Sources d'information & 7 & $\begin{array}{l}\text { Décrire toutes les sources d'information ( } p . \text { ex. bases de données } \\
\text { avec les dates de la couverture, les coordonnées des auteurs de } \\
\text { l'étude pour déterminer d'autres études) dans la recherche et la date } \\
\text { de la dernière recherche. }\end{array}$ \\
\hline Recherche & 8 & $\begin{array}{l}\text { Présenter une version électronique intégrale de la stratégie de } \\
\text { recherche pour au moins une base de données, y compris les limites } \\
\text { utilisées, de façon à pouvoir la reproduire. }\end{array}$ \\
\hline Sélection de l'étude & 9 & $\begin{array}{l}\text { Expliquer le processus de sélection des études (p. ex. le tri, } \\
\text { l'admissibilité, l'inclusion dans l'examen systématique, et s'il y a lieu, } \\
\text { inclusion dans la méta-analyse). }\end{array}$ \\
\hline $\begin{array}{l}\text { Processus de collecte } \\
\text { des données }\end{array}$ & 10 & $\begin{array}{l}\text { Décrire la méthode d'extraction des données à partir des rapports (p. } \\
\text { ex. formulaires pilotes, indépendante, en double) et tout processus } \\
\text { visant l'obtention et la confirmation des données auprès des } \\
\text { chercheurs. }\end{array}$ \\
\hline Éléments de données & 11 & $\begin{array}{l}\text { Citer et définir toutes les variables pour lesquelles on cherchait des } \\
\text { données ( } p \text {. ex. le projet PICOS, les sources de financement) et pour } \\
\text { lesquelles on a fait des suppositions et apporté des simplifications. }\end{array}$ \\
\hline $\begin{array}{l}\text { Risque de biais dans } \\
\text { chaque étude }\end{array}$ & 12 & $\begin{array}{l}\text { Décrire les méthodes utilisées pour évaluer le risque de biais de } \\
\text { chacune des études (y compris la spécification du niveau de } \\
\text { l'évaluation, c.-à-d. au niveau de l'étude ou des résultats) et la façon } \\
\text { dont ces renseignements doivent être utilisés pour une synthèse des } \\
\text { données. }\end{array}$ \\
\hline Mesures sommaires & 13 & $\begin{array}{l}\text { Énoncer les principales mesures sommaires (p. ex. le risque relatif, } \\
\text { les écarts dans les moyens). }\end{array}$ \\
\hline Synthèse des résultats & 14 & $\begin{array}{l}\text { Décrire les méthodes de manipulation des données et de } \\
\text { combinaison des résultats des études, si c'est fait, y compris les } \\
\text { mesures d'uniformité (p. ex. 12) pour chaque méta-analyse. }\end{array}$ \\
\hline $\begin{array}{l}\text { Risque de biais dans } \\
\text { l'ensemble des études }\end{array}$ & 15 & $\begin{array}{l}\text { Mentionner toute évaluation du risque de biais pouvant avoir une } \\
\text { incidence sur les preuves cumulatives (p. ex. biais de publication, } \\
\text { rapports sélectifs au sein des études). }\end{array}$ \\
\hline $\begin{array}{l}\text { Analyses } \\
\text { supplémentaires }\end{array}$ & 16 & $\begin{array}{l}\text { Décrire les méthodes d'analyses supplémentaires (p. ex. analyses de } \\
\text { la sensibilité ou des sous-groupes, méta-régression), et si c'est fait, } \\
\text { indiquer les méthodes prédéterminées. }\end{array}$ \\
\hline \multicolumn{3}{|l|}{ Résultats } \\
\hline Sélection de l'étude & 17 & $\begin{array}{l}\text { Fournir le nombre d'études présélectionnées, évaluées aux fins } \\
\text { d'admissibilité et incluses dans l'examen, avec les raisons de } \\
\text { l'exclusion à chaque étape, idéalement avec un organigramme. }\end{array}$ \\
\hline $\begin{array}{l}\text { Caractéristiques de } \\
\text { l'étude }\end{array}$ & 18 & $\begin{array}{l}\text { Pour chaque étude, présenter les caractéristiques pour lesquelles des } \\
\text { données ont été extraites (p. ex. la taille de l'étude, le projet PICOS, } \\
\text { la période de suivi) et fournir les citations. }\end{array}$ \\
\hline Risque de biais au sein & 19 & Présenter les données sur le risque de biais de chaque étude, et si \\
\hline
\end{tabular}




\begin{tabular}{|c|c|c|}
\hline des études & & $\begin{array}{l}\text { elle est disponible, une évaluation du niveau de résultats (voir le point } \\
\text { 12). }\end{array}$ \\
\hline $\begin{array}{l}\text { Résultats d'études } \\
\text { individuelles }\end{array}$ & 20 & $\begin{array}{l}\text { Pour tous les résultats pris en compte (avantages ou préjudices), } \\
\text { présenter pour chaque étude des données sommaires simples pour } \\
\text { chaque groupe d'intervention et les estimations des effets et les } \\
\text { intervalles de confiance, idéalement avec un graphique en forêt. }\end{array}$ \\
\hline Synthèse des résultats & 21 & $\begin{array}{l}\text { Présenter les principaux résultats de l'examen. Si des méta-analyses } \\
\text { sont réalisées, inclure pour chacune des intervalles de confiance et } \\
\text { des mesures d'uniformité. }\end{array}$ \\
\hline $\begin{array}{l}\text { Risque de biais dans } \\
\text { l'ensemble des études }\end{array}$ & 22 & $\begin{array}{l}\text { Présenter les résultats de l'évaluation du risque de biais dans } \\
\text { l'ensemble des études (voir le point 15). }\end{array}$ \\
\hline $\begin{array}{l}\text { Analyses } \\
\text { supplémentaires }\end{array}$ & 23 & $\begin{array}{l}\text { Fournir les résultats des analyses supplémentaires [p. ex. analyses } \\
\text { de la sensibilité ou des sous-groupes, méta-régression (voir le point } \\
\text { 16)]. }\end{array}$ \\
\hline \multicolumn{3}{|l|}{ Discussion } \\
\hline $\begin{array}{l}\text { Résumé des données } \\
\text { probantes }\end{array}$ & 24 & $\begin{array}{l}\text { Résumer les principales constatations, y compris la force des } \\
\text { données probantes pour chaque résultat principal; tenir compte de } \\
\text { leur pertinence par rapport aux groupes clés (p. ex. les fournisseurs } \\
\text { de soins de santé, les utilisateurs et les responsables des politiques). }\end{array}$ \\
\hline Limites & 25 & $\begin{array}{l}\text { Discuter des limites au niveau de l'étude et des résultats (p. ex. le } \\
\text { risque de biais), ainsi qu'au niveau de l'examen (p. ex. récupération } \\
\text { incomplète des recherches définies, biais de déclaration). }\end{array}$ \\
\hline Conclusion & 26 & $\begin{array}{l}\text { Donner une interprétation générale des résultats dans le contexte } \\
\text { d'autres données probantes et implications pour les futures } \\
\text { recherches. }\end{array}$ \\
\hline \multicolumn{3}{|l|}{ Financement } \\
\hline Financement & 27 & $\begin{array}{l}\text { Décrire les sources de financement de l'examen systématique et } \\
\text { d'autres services de soutien ( } p \text {. ex. l'approvisionnement de données) } \\
\text { et le rôle des bailleurs de fonds pour l'examen systématique. }\end{array}$ \\
\hline
\end{tabular}

\footnotetext{
${ }^{1} \mathrm{~N}^{0}$ : numéro

${ }^{2}$ La description du résumé a été modifiée pour le Relevé des maladies transmissibles au Canada (RMTC).

${ }^{3}$ Reflète la correction telle qu'elle est indiquée sur le site http://www.prisma-statement.org/statement.htm.
} 
Figure 1 : Organigramme PRISMA de 2009

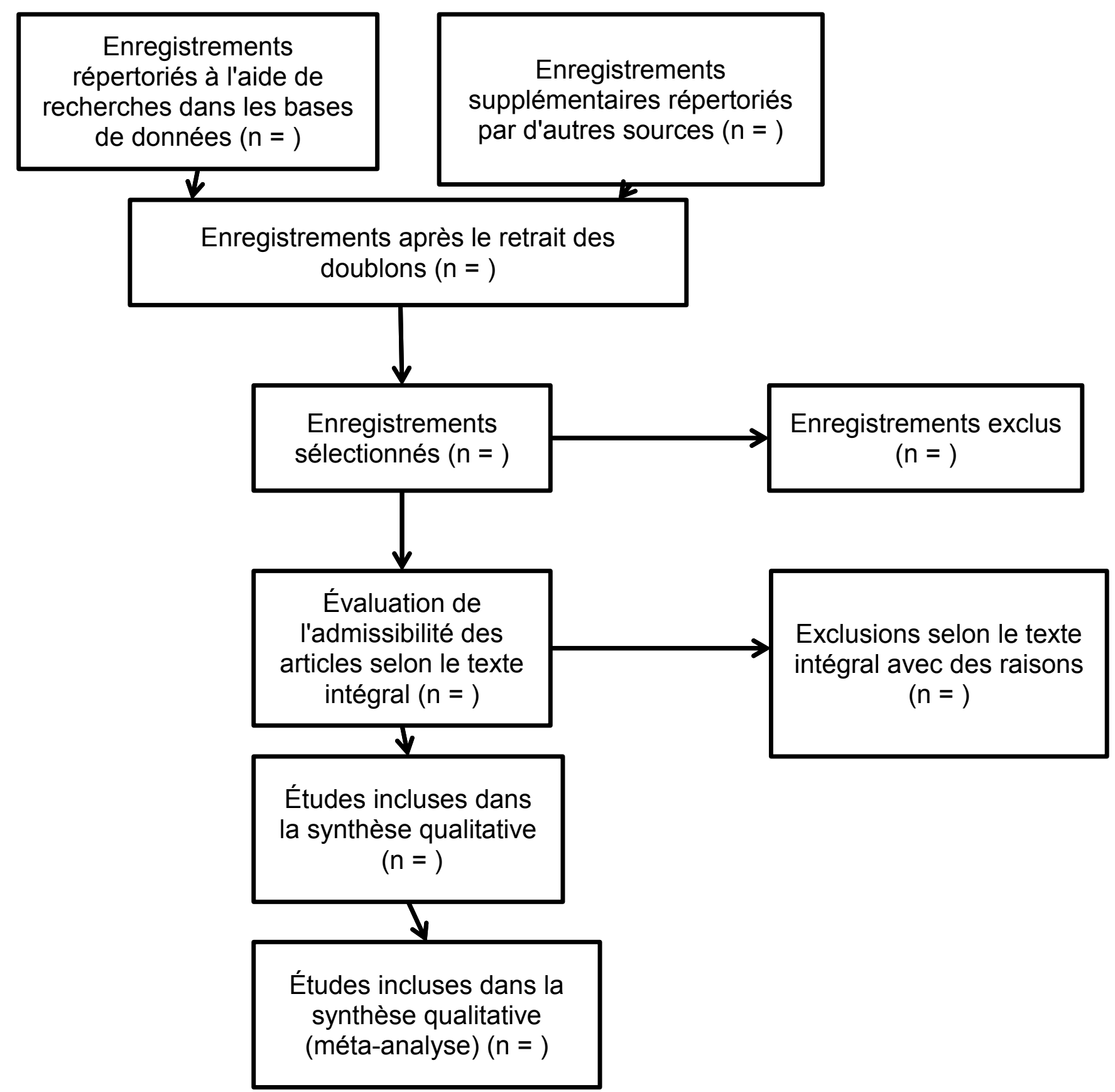




\section{Références}

(1) Moher D, Liberati A, Tetzlaff J, Altman DG, for the PRISMA Group. Preferred reporting items for systematic reviews and meta-analyses: The PRISMA statement. BMJ. 2009;339:332-336.

(2) Welch V, Petticrew M, Tugwell P, Moher D, O'Neill J, Waters E, White H, PRISMA-Equity Bellagio Group. PRISMAequity 2012 extension: Reporting guidelines for systematic reviews with a focus on health equity. PLoS Med. 2012;9(10):e1001333.

(3) Beller EM, Glasziou PP, Altman DG, Hopewell S, Bastian H, Chalmers I, Gøtzsche PC, Lasserson T, Tovey D, PRISMA for Abstracts Group. PRISMA for abstracts: Reporting systematic reviews in journal and conference abstracts. PLoS Med. 2013;10(4):e1001419.

(4) Moher D, Shamseer L, Clarke M, Ghersi D, Liberati A, Petticrew M, Shekelle P, Stewart LA. Preferred reporting items for systematic review and meta-analysis protocols (PRISMA-P) 2015 statement. Syst Rev. 2015;4(1):1. 